\title{
CONFINED-CHALCOGENIDE PHASE CHANGE MEMORY WITH THIN METAL INTERLAYER FOR LOW RESET CURRENT BY FINITE ELEMENT MODELING
}

\author{
S. Harnsoongnoen ${ }^{1, a}$, C. Sa-ngiamsak ${ }^{1,2, b,{ }^{*}}$ and A. Siritaratiwat ${ }^{1,2, c}$ \\ ${ }^{1}$ Department of Electrical Engineering, Khon Kaen University, Khon Kaen, 40002, Thailand \\ ${ }^{2}$ Industry/University Cooperation Research Center in HDD Component, Faculty of Engineering, \\ Khon Kaen, 40002, Thailand \\ a5070400157@stdmail.kku.ac.th, ${ }^{\text {b, }}{ }^{*}$ corresponding author: chiranut@kku.ac.th, ${ }^{c}$ apirat@kku.ac.th
}

Keywords: Phase change memory (PCM), Confined-chalcogenide phase change memory with thin metal interlayer (CCTMI), Normal bottom contact (NBC), Low reset current, Finite element.

\begin{abstract}
This paper reports on the confined-chalcogenide phase change memory with thin metal interlayer (CCTMI) with the operating reset current of $0.6 \mathrm{~mA}-30 \mathrm{~ns}$. This cell offers low reset current with simple architecture and fabrication. Thermal and heat flux distribution of both the normal-bottom-contact (NBC) and a proposed CCTMI PCM cells were carefully analyzed and simulated by two-dimensional finite element modeling. It is intriguingly found that the reset operation current of the CCTMI cell is $44 \%$ lower than that of the NBC. CCTMI has capability to solve an over-programming fail issue due to confined heat dissipation in active area.
\end{abstract}

\section{Introduction}

Recently, phase change memory (PCM) has been considered an one of the best candidates for nextgeneration nonvolatile memories (NVMs) due to its better properties comparing with previous conventional NVMs such as fast access time, low power consumption, low cost, high endurance, high scalability, and good data retention [1,2]. The principle of PCM is on the basis of the ability of chalcogenides (e.g. $\mathrm{Ge}_{2} \mathrm{Sb}_{2} \mathrm{Te}_{5}$ : GST) to be reversibly structure transformed between highly resistive amorphous and lowly resistive crystalline [3]. Nevertheless, the setback of PCM is a high reset current, for instance, is almost 1-2mA [4]. Nowadays, most of efforts have been made upon decreasing the programming power of the PCM cell. There are two most effective methods to reduce the reset current: one is to optimize the properties of material [5] and improve the structure of PCM $[6,7]$. However, the fabrication of both approaches is complicated and the area of these cells is larger than that of the corresponding normal bottom contact (NBC) cell. To achieve a low reset current with simple structure, the confined-chalcogenide (CC) cell is proposed by Yin et al. [8]. The reset operation of a CC cell is achievable at $0.8 \mathrm{~mA}$. Further reduction of a reset current can be attained by setting a chalcogenide material with much higher resistivity than a resistive heater [9]. Optimisation of a reset current and to solve the over-programming fail of this case, PCM with thin metal inserted chalcogenide (bottom chalcogenide/metal interlayer/top chalcogenide) structure has been proposed [10]. Nevertheless, reports on the combination of confined and metal inserted PCM structure have never been published. Therefore, this work reports on the confinedchalcogenide phase change memory with thin metal interlayer (CCTMI) with the operating reset current using finite element method. The comparison of the PCM performance between the proposed CCTMI cell with a NBC cell by using finite element modeling is discussed.

\section{PCM cell structures}

The phase change film with thin metal inserted structure has been proposed to realize memory storage in PCM. 


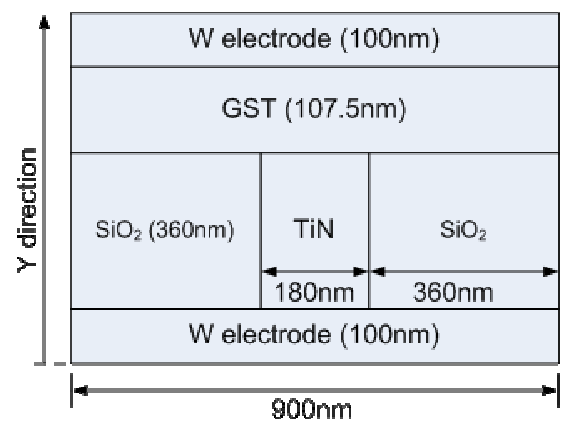

(a)

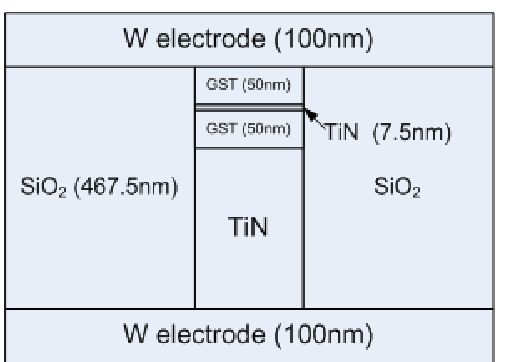

(b)

Fig. 1.Schematic cross-sections diagram of PCM cell structure for simulation (a) NBC cell and (b) CCTMI cell.

Fig. 1(a) and 1(b) show the geometries of the NBC and CCTMI cells used for simulations. $\mathrm{W}$ is used as top and bottom electrode, phase change material is $\mathrm{Ge}_{2} \mathrm{Sb}_{2} \mathrm{Te}_{5}$, inserted metal is $\mathrm{TiN}$, heater and the dielectric are used $\mathrm{TiN}$ and $\mathrm{SiO}_{2}$, respectively. The material properties are listed in Table 1.

\section{Thermal and electrical models}

The drastic resistance variation of PCM is attributed to phase-change triggered by Joule heating which occurs when the current flows through the cell. The mathematical model of heat transfer is:

$$
d C \frac{\partial T}{\partial t}-\nabla \cdot(k \nabla T)=Q
$$

where $d$ is the density, $\mathrm{C}$ is the heat capacity, $\mathrm{T}$ is the temperature, $\mathrm{k}$ is the thermal conductivity, $\mathrm{t}$ is the time and $\mathrm{Q}$ is the heat flux. The heat generated by Joule heating $\mathrm{Q}$ is given by

$$
Q=\frac{1}{\sigma}|J|^{2}=\sigma|\nabla V|^{2}
$$

where $\sigma$ is the electric conductivity, $\mathrm{J}$ is the electric current density and $\mathrm{V}$ is the electric potential. Assumptions for this work are (a) all of the exterior boundaries are set at room temperature (298K) whereas those of interior boundaries are set as having continuous boundary conditions, (b) junction temperature $T_{j}$ reaches $353 \mathrm{~K}$ at $3.3 \mathrm{~V}$ or $5 \mathrm{~V}$ of operation voltage and the top $\mathrm{W}$ layer remains at room temperature, (c) the thermal and electrical properties of the material have no temperature dependence. Thermal and electrical properties of materials related in the simulation are given in Table 1.

Table 1. Physical properties of materials used in simulations [9]

\begin{tabular}{|l|c|c|c|c|}
\hline \multicolumn{1}{|c|}{ Material } & $\begin{array}{r}\text { Mass density } \\
d\left(\mathrm{~kg} / \mathrm{m}^{2}\right.\end{array}$ & $\begin{array}{r}\text { Specific heat } \\
C(\mathrm{~J} / \mathrm{kgK}\end{array}$ & $\begin{array}{c}\text { Thermal conductivity } \\
k(\mathrm{~W} / \mathrm{mK})\end{array}$ & $\begin{array}{c}\text { Resistivity } \\
\rho_{e}(\Omega m)\end{array}$ \\
\hline $\mathrm{GST}_{\text {bottom/top }}$ & 6200 & 202 & 0.46 & $1 \times 10^{-3}$ \\
\hline $\mathrm{TiN}_{\text {insert }}$ & 5240 & 784 & 0.44 & $1 \times 10^{-2}[11]$ \\
\hline $\mathrm{TiN}_{\text {heater }}$ & 5240 & 784 & 0.44 & $1.2 \times 10^{-4}$ \\
\hline $\mathrm{W}$ & 19300 & 132 & 174 & $5.39 \times 10^{-8}$ \\
\hline $\mathrm{SiO}_{2}$ & 2330 & 1330 & 1.4 & $1 \times 10^{14}$ \\
\hline
\end{tabular}

\section{Results and Discussions}

A requirement of GST layer is to generate heat with temperature higher than the melting temperature of GST (Tm), 905K, in order to operate during a reset mode (amorphous state). A programming current pulse with amplitude of $0.6 \mathrm{~mA}$ and width of $30 \mathrm{~ns}$ was fed to both NBC and 
CCTMI cells. The gradient of temperature is indicated in red scale level. Direction and heat flux density are defined as arrows. Level of brightness indicates the level of temperature.

The comparison of the temperature profile and heat flux distribution of both cells is shown in Fig. 2(a) and 2(b). It reveals that CCTMI cell has less heat loss in passive area than that NBC due to the resistivity of $\mathrm{W}$ is very small $(53.9 \mathrm{n} \Omega . \mathrm{m})$, but its thermal conductivity $(174 \mathrm{~W} / \mathrm{mK})$ is much larger than GST $(0.46 \mathrm{~W} / \mathrm{mK})$. The high thermal conductivity of $\mathrm{W}$ causes large heat dissipation through the $\mathrm{W}$ electrode. However, the CCTMI is inserted by $\mathrm{TiN}_{\text {insert }}$ with low thermal conductivity and high resistivity, this will suppress the heat diffusion from the GST to $\mathrm{W}$ and the maximum temperature in CCTMI is almost located at the middle of the $\mathrm{TiN}_{\text {insert }}$ because of the resistivity of $\operatorname{TiN}_{\text {insert }}(10 \mathrm{~m} \Omega . \mathrm{m})$ is more than of GST $(1 \mathrm{~m} \Omega . \mathrm{m})$. Therefore, the temperature increasing of GST is caused by both thermal transfer and self-Joule-heating in GST. Moreover, the shape of heat distribution in an active area of the CCTMI cell is in eclipse and homogeneous pattern while NBC has half-eclipse and wider heat distributed area. This implies the better performance of CCTMI over NBC in term of heat jet direction and uniformity of heat distribution of the cell hence higher temperature at the active area of CCTMI cell.

Further investigation of the thermal distribution of both structures was conducted by plotting the temperature distribution along the y-axis as shown in Fig. 3(a). Simulations show that the maximum temperature of the GST region of the CCTMI cell at a reset current of $0.6 \mathrm{~mA}-30 \mathrm{~ns}$ is above Tm of GST while that of NBC cell is much lower and does not reach to Tm of GST. Fig. 3(b) shows the temperature change at the middle GST layer in NBC cell and in interface between $\mathrm{GST}_{\text {bottom }} / \mathrm{TiN}_{\text {insert }}$ in CCTMI cell. To more clearly illustrate the temperature raise linearly and then start to saturate during the on-current period and then drop dramatically fast during the off-current period. Moreover, it reveals a fast and sharper temperature ramping up and down of the CCTMI cell. This signifies a superior performance of the CCTMI cell over NBC cell in term of shorter reset time and higher temperature under the same level of biasing current, a reset current of $0.6 \mathrm{~mA}$; hence, lower-power consumption PCM is a promise of this CCTMI cell.

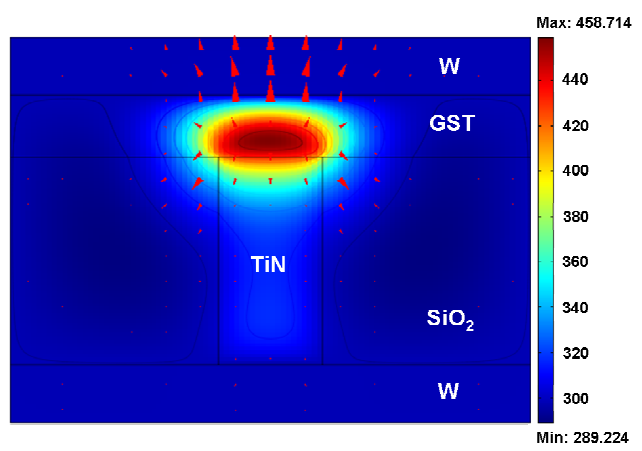

(a)

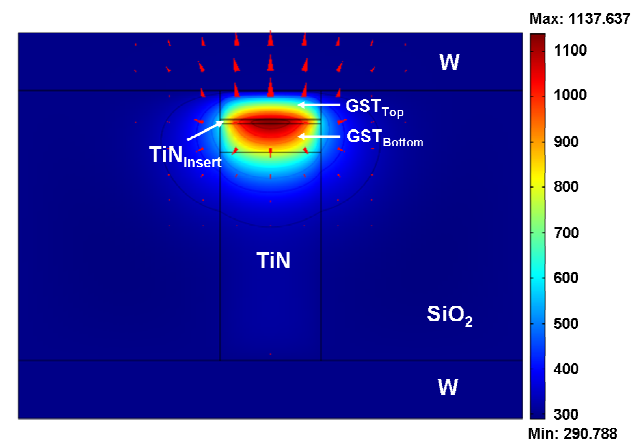

(b)

Fig. 2. Thermal and heat flux distribution of PCM cell at reset operation pulse of $0.6 \mathrm{~mA}-30 \mathrm{~ns}$ : (a) NBC cell (b) CCTMI cell.

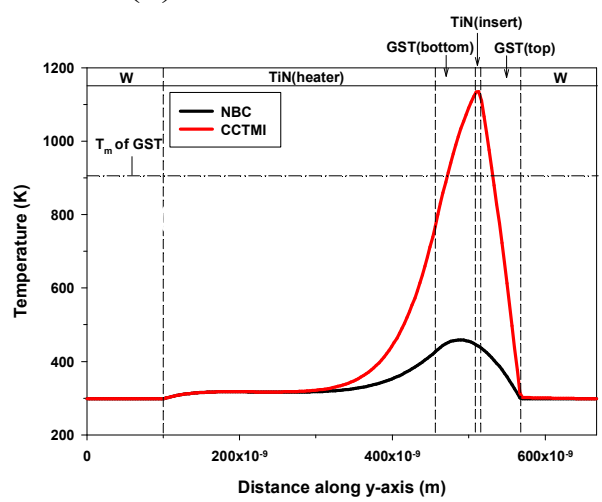

(a)

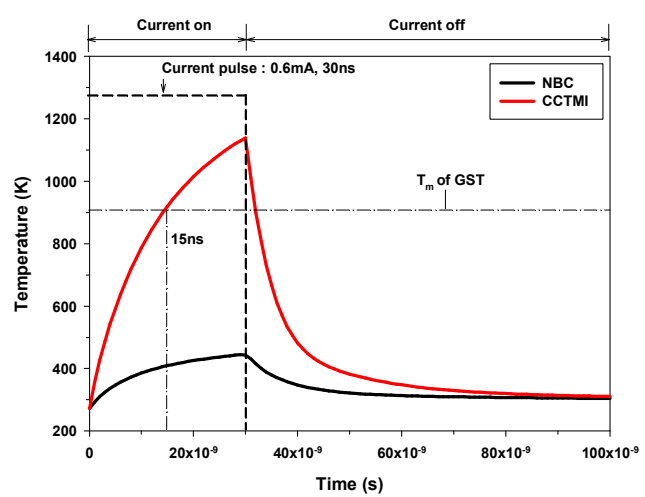

(b)

Fig. 3. Temperature profiles for NBC and CCTMI cells (a) along y-axis (b) position in GST when applying a pulse of $0.6 \mathrm{~mA}-30 \mathrm{~ns}$. 
Details of melted GST layer of both cells with various reset currents are carefully investigated in order to monitor an optimized programming reset current where a stable resetamorphous state is still achievable. Further investigation of suitable amplitude of a reset current for NBC cell was carried by varying a reset current applied to NBC cell starting from 0.1 to $1 \mathrm{~mA}$ with

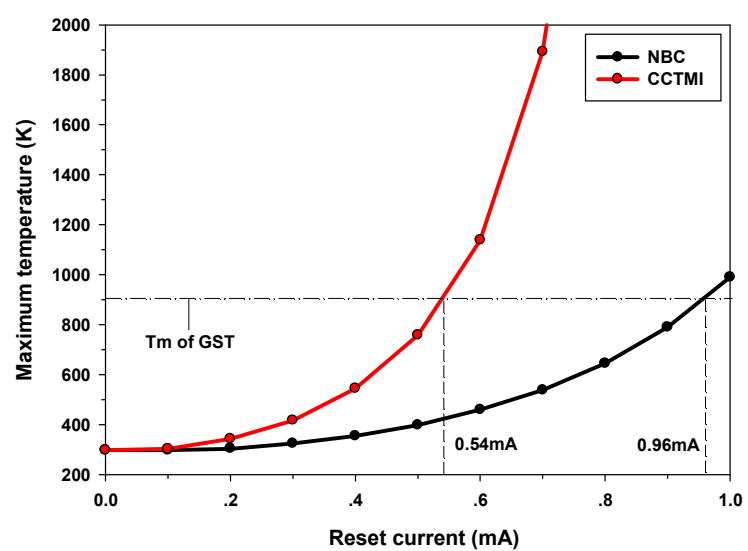
the current step of $0.1 \mathrm{~mA}$ and a pulse width of $30 \mathrm{~ns}$. The maximum temperature of the cell with various current amplitude was plotted in Fig. 4. It reveals that to reach the melting temperature of GST during the reset state of PCM, a good stable amorphous state of NBC cell needs the reset current over $0.9 \mathrm{~mA}$ while CCTMI cell requires slightly below $0.6 \mathrm{~mA}$.

Fig. 4. Maximum temperature versus reset current and a width of $30 \mathrm{~ns}$.

\section{Conclusions}

The simulation results of this work conclude that:

(a) The CCTMI cell generates heat at the GST layer at almost 3 times higher than the NBC cell with the same reset current amplitude of $0.6 \mathrm{~mA}$ and the width of $30 \mathrm{~ns}$.

(b) The CCTMI structure can offer a reduction of the NBC reset current by $44 \%$.

(c) The CCTMI has less heat loss and better controlled of heat flux density over the active area. Therefore, the problem of over-programming fail issue is reduced.

This implies the high-speed and low-power consumption CCTMI cell structure that can hold a promise as a future technology as memory devices.

\section{Acknowledgments}

The authors would like to thank the Computer Research and Service Center, King Mongkut's Institute of Technology Ladkrabang for program support. This work is supported by I/U CRC in HDD Components, Faculty of Engineering, Khon Kaen University (CPN-R\&D-01-08-51).

\section{References}

[1] R.Y. Kim, H.G. Kim and S.G. Yoon: Appl. Phys. Lett. Vol. 89 (2006), p. 102107.

[2] S. Lai and T. Lowrey: IEDM Tech. Dig., (2003), p. 803.

[3] S.R. Ovshinsky: Phys. Rev. Lett. Vol. 21 (1968), p. 1450.

[4] D.H. Kang and et al.: J. Appl. Phys. Vol. 100 (2006), p. 054506.

[5] T. Zhang and et al.: Appl. Phys. Lett. Vol. 91 (2007), p. 222102.

[6] Y.H. Ha and et al.: Symp. VLSI Technology Dig. Tech. Pap. (2003), p. 175.

[7] F. Pellizzer and et al.: Symp. VLSI Technolgy Dig. Tech. Pap. (2004), p. 18.

[8] Y.Yin, H. Sone and S. Hosaka: Jpn. J. Appl. Phys. Vol. 45 (2006), p. 6177.

[9] Y.Yin, H. Sone and S. Hosaka: Jpn. J. Appl. Phys. Vol. 45 (2006), p. 8600.

[10] J.H. Yi and et al.: IEDM Tech. Dig., (2003), p. 37.3.1.

[11] D. Choi and P.N. Kumta: J. Am. Ceram. Soc. Vol. 88 (2005), p. 2030. 